\title{
Alterações biomecânicas da córnea porcina com riboflavina fotossensibilizada por luz não-ultravioleta
}

\author{
Biomechanical changes on porcine corneas after riboflavin \\ induced crosslink technique with non-UV light
}

Gustavo Teixeira Grottone', Fábio Suga², Daniela Matsuda³, Luciane Lopes Vaccaro, Juliana de França Teixeira Grottone $^{5}$, João Carlos Grottone

\section{Resumo}

Objetivo: Demonstrar a possibilidade de alterar a plasticidade do estroma corneano através da utilização do agente fotossensível riboflavina associado ao uso de iluminação não-ultravioleta. Métodos: Experimento prospectivo duplo cego. Vinte e cinco olhos de porcos enucleados até 24 horas antes do experimento, foram divididos nos seguintes grupos: Grupo RB01+L-Riboflavina 0,1\% com irradiação de luz azul; Grupo RB01Riboflavina 0,1\% sem irradiação de luz azul; Grupo RB05+L-Riboflavina 0,5\% com irradiação de luz azul; Grupo RB05-Riboflavina 0,5\% sem irradiação; Grupo L-Solução salina balanceada e irradiação de luz azul. Resultados: Após o tratamento das informações dos grupos estudados, obtivemos diferenças estatisticamente significantes no grupos RB01+L e RB05+L(p<0,05). Os grupos sem irradiação de luz azul e o grupo somente com irradiação da luz azul sem riboflavina, não apresentaram diferenças estatisticamente significantes. Conclusão: A utilização de riboflavina para efetuar o processo de aumento de ligações covalentes entre as fibrilas colágenas pode ser uma das chaves para o controle de doenças da córnea como o ceratocone. Nas concentrações estudadas e doses de irradiância concebidas, o processo de aumento das características biomecânicas da córnea foram obtidas com sucesso.

Descritores: Riboflavina/farmacologia; Agentes fotossensibilizantes/farmacologia; Substância própria/efeitos de drogas; Fotoquímica;Biomecânica; Fotodegradação; Raios ultravioleta;Porco

\footnotetext{
${ }^{1}$ Mestre, Chefe do Setor de Catarata e Bioengenharia da Santa Casa de Misericórdia de Santos (SP), Brasil;

${ }^{2}$ Preceptor, Médico Assistente do Serviço de Oftalmologia da Santa Casa de Misericórdia de Santos (SP), Brasil;

${ }^{3}$ Residente do Serviço de Oftalmologia da Santa Casa de Misericórdia de Santos (SP), Brasil;

${ }_{5}^{4}$ Acadêmica da Faculdade de Ciências Médicas de Santos (SP), Brasil;

${ }^{5}$ Acadêmica da Universidade Metropolitana de Santos - UNIMES - Santos (SP), Brasil.

${ }^{6}$ Professor Titular da Disciplina de Oftalmologia da Faculdade de Ciências Médicas de Santos; Chefe do Serviço de Oftalmologia da Santa Casa de Misericórdia de Santos (SP), Brasil.
}

Trabalho realizado no Serviço de Oftalmologia da Santa Casa de Misericórdia de Santos

Recebido para publicação em: 20/2/2008 - Aceito para publicação em 16/6/2008 


\section{INTRODUÇÃO}

D evido à ectasia corneana causada por doenças como o ceratocone, ectasia pós-cirurgia refrativa e outras doenças que levam à mudança na estrutura e arquitetura corneana, muitos pacientes vem sendo submetidos a vários procedimentos cirúrgicos. Dentre as tentativas para conter a progressão ou pelo menos viabilizar uma melhora na acuidade visual, temos métodos como o implante de anel intraestromal e a cirurgia fotorrefrativa. Entretanto, tais métodos se mostram insuficientes para conter a doença e em muitos casos podem inclusive agravar as características instáveis de tais córneas.

Desta maneira, fica claro que somente um procedimento que cause mudanças biomecânicas na córnea, poderia causar um efeito mais duradouro nestes olhos. A técnica de ligações covalentes das fibras de colágeno "cross-linking" no estroma corneano, pode ser uma solução eficiente no controle destes pacientes pelo período de até 3 anos. Tal efeito transitório ocorre devido ao processo de substituição das fibras colágenas no organismo ${ }^{(1)}$.

Através da informação obtida em estudos preliminares, a riboflavina é uma substância que pode ser usada de maneira criteriosa para alcançar resultados benéficos na estabilização corneana. O aumento de $70 \%$ da estabilidade biomecânica da córnea já foi verificado em estudos experimentais, utilizando a riboflavina $0,1 \%{ }^{2}$.

A riboflavina é uma substância fotossensível que ao ser irradiada libera radicais livres, aumentando o número de ligações covalentes entre as fibrilas colágenas, promovendo uma maior resistência mecânica na córnea e por sua vez uma menor propensão a alterações de estrutura e curvatura, tanto em córneas porcinas quanto humanas ${ }^{(3)}$ (Figura 1$)$.

Segundo outro estudo, a área mais afetada pelo tratamento com a riboflavina e irradiação ultravioleta é a porção anterior do estroma corneano. Cerca de $70 \%$ da irradiação ultravioleta se confina nas primeiras 200 micras da córnea ${ }^{(4)}$.

O processo de "cross-linking" já é conhecido dos bioquímicos através da glicolização das fibrilas de colágeno, fato este que corrobora a ausência de casos de ceratocone em pacientes previamente diabéticos. Devido aos altos níveis de glicose, ocorre a glicolização das fibrilas de colágeno ${ }^{(5)}$.

Experimentalmente, observou-se um aumento do diâmetro das fibras colágenas corneanas. Ao exame de microscopia eletrônica com aumento de 98.000 vezes foi possível visualizar um aumento de $11 \%$ no diâmetro

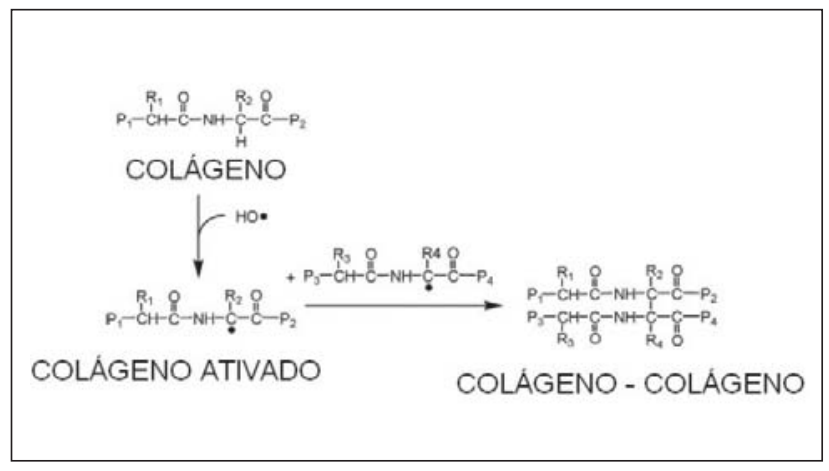

Figura 1: Processo de hidroxilação por radicais livres e ligações covalentes entre fibrilas colágenas

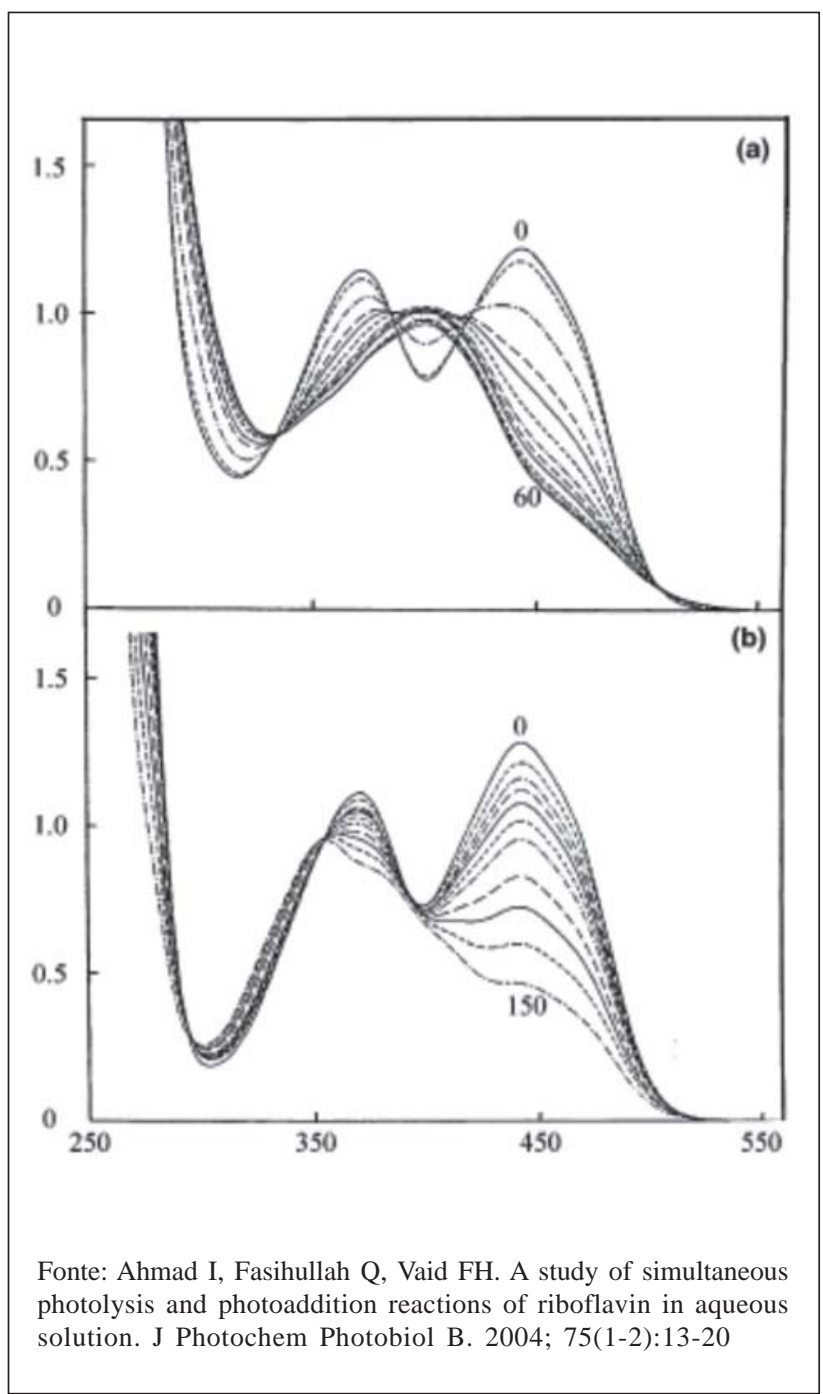

Figura 2: Variação da absorção de luz pela riboflavina de acordo com o espectro e concentração. A - Em solução tampão-fosfato 2,00 M; B - Em solução tampão-fosfato 0,05 M 


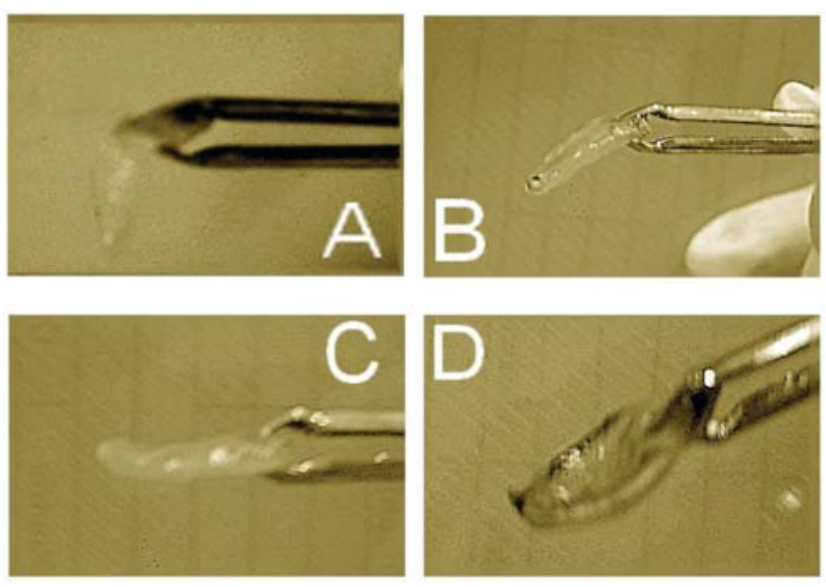

Figura 3: Classificação do teste biomecânico. A - Resultado insuficiente; B - Resultado fraco; C - Resultado razoável e D - Resultado forte

das fibras colágenas. Apesar da fibrilas não serem visualizáveis nem com microscopia eletrônica, uma das evidências indiretas foi o aumento da resistência biomecânica do estroma anterior das córneas examinadas $^{6}$. Outro efeito demonstrado neste tipo de tratamento foi o aumento da resistência à digestão enzimática ${ }^{(7)}$.

O tratamento proposto até o momento na literatura utiliza radiação ultravioleta para propiciar os mecanismos de fotodegradação da riboflavina ${ }^{\left({ }^{8}\right)}$. Entretanto, a toxicidade da luz ultravioleta para a córnea e mais especificamente para o endotélio corneano e cristalino, tem um limiar muito baixo, sendo inseguro o controle efetivo desta irradiação pelo protótipos atuais ${ }^{(9)}$.

Conforme outros estudos encontrados na literatura, a excitação da riboflavina por luz de espectro diferente da ultravioleta é possível. A riboflavina apresenta dois picos máximos de absorção luminosa. Um deles é o ultravioleta no comprimento de onda de $365 \mathrm{~nm}$ (Figura 2). O outro encontra-se no espectro azul entre $435 \mathrm{~nm}$ e $450 \mathrm{~nm}$ de comprimento de onda ${ }^{(10)}$ :

A) Em solução tampão-fosfato 2,00 M;

B) Em solução tampão-fosfato 0,05 M.

Devido ao caráter menos agressivo para a córnea do comprimento de onda da luz azul, este vem a ser uma nova opção de tratamento para esta modalidade de terapia fotodinâmica.

Conforme a literatura nacional é possível utilizar olhos de origem porcina para avaliar resultados de tratamentos baseados em remodelação da córnea ${ }^{(11)}$.

Este trabalho tem como objetivo demonstrar a possibilidade de alterar a plasticidade do estroma corneano através da utilização do agente fotossensível riboflavina associado ao uso de iluminação nãoultravioleta.

\section{Métodos}

Vinte e cinco olhos de porcos enucleados até 24 horas antes do experimento foram divididos aleatoriamente e igualmente nos seguintes grupos: Grupo RB01+LRiboflavina 0,1\% com irradiação de luz azul; Grupo RB01Riboflavina $0,1 \%$ sem irradiação de luz azul;Grupo RB05+L-Riboflavina 0,5\% com irradiação de luz azul; Grupo RB05-Riboflavina 0,5\% sem irradiação; Grupo LSolução salina balanceada e irradiação de luz azul.

Para a irradiação dos olhos em estudo foi desenvolvido protótipo em nosso setor de bioengenharia utilizando iluminação por LED. As características da luz azul (Luxeon-Philips/USA) seguirão os parâmetros pré-estudados, sendo eles os seguintes: comprimento de onda da luz visível de $450 \mathrm{~nm}$, com irradiância de $3 \mathrm{~mW} / \mathrm{cm} 2$, área de tratamento de $7 \mathrm{~mm}$, com distância do ápice corneano de $10 \mathrm{~mm}$.

A instilação de riboflavina foi iniciada 5 minutos antes da irradiação (assim como a solução de soro fisiológico para os grupos sem irradiação). Após iniciada a irradiação, pausas entre cada 5 minutos de aplicação foram feitas para nova instilação da substância estudada até que se completasse 30 minutos do início da irradiação.

Após o término dos experimentos, os olhos foram removidos da solução salina balanceada e submetidos à ceratectomia penetrante para avaliação biomecânica. As córneas removidas foram submetidas ao teste biomecânico de inspecção após o mascaramento e distribuição randômica das amostras por pesquisador do grupo não envolvido no processo da ceratectomia. Tal teste consiste em fixar com uma pinça no quartil externo, a córnea em posição horizontal com o ápice voltado inferiormente, durante o período de 120 segundos. Após esse período, foi verificada a manutenção ou não do posicionamento corneano na posição inicial horizontal.

O resultado do teste biomecânico de inspeção foi regido pela seguinte classificação: A - Resultado insuficiente (o ângulo entre a pinça e a córnea é maior que 45 graus); B - Resultado fraco (o ângulo entre a pinça e a córnea é maior que 15 graus e menor que 45 graus); C - Resultado razoável (o ângulo entre a pinça e a córnea é menor que 15 graus, mas a córnea não mantém sua concavidade para cima); D - Resultado forte (o ângulo entre a pinça e a córnea é menor que 15 graus e a córnea mantém sua concavidade para cima) (Figura 3).

Os resultados foram agrupados e tratados com o programa de estatística NCSS. Foram utilizados o teste de Kruskal-Wallis (com $\mathrm{p}<0,05)$ e teste de Bonferroni para avaliar a significância estatística dos resultados. 


\section{Resultados}

Após o tratamento das informações dos grupos estudados (Tabela 1), obtivemos diferenças estatisticamente significantes no grupos $\mathrm{RB} 01+\mathrm{L}$ e $\mathrm{RB} 05+\mathrm{L}(\mathrm{p}<0,05)$ (Tabela 2). No grupo RB01+L, a mediana dos resultados foi igual a classificação $\mathrm{C}$ (resultado razoável). O grupo RB05+L obteve os melhores resultados com mediana igual a classificação D (resultado forte). Os grupos RB01e RB05 obtiveram mediana igual a classificação B. Já no grupo L em que não se utilizou riboflavina, o resultado obtido em todas as amostras foi igual a classificação A. Ainda foi constatado que os grupos $\mathrm{RB} 01+\mathrm{L}$ e $\mathrm{RB} 05+\mathrm{L}$ apresentaram diferenças estatisticamente significantes com $\mathrm{p}<0,05$. Os grupos RB01 e RB05(sem irradiação de luz azul) e o grupo L, não apresentaram diferenças estatisticamente significantes.

\section{DıscuSSÃo}

Conforme previsto em trabalhos anteriores, a irradiação da riboflavina por fonte luminosa foi capaz de causar alterações biomecânicas no estroma corneano ${ }^{(2-6)}$. De acordo com trabalhos relacionados com a bioquímica de alimentos, a luz visível não ultravioleta é capaz de degradar a riboflavina e subprodutos com a geração de radicais livres oxidantes ${ }^{(8,10)}$. O espectro de luz azul demonstrou-se capaz de afetar com suficiente absorção a riboflavina, causando o provável aumento de ligações entre proteínas corneanas como o colágeno.

O grupo em que somente a luz azul foi irradiada, não demonstrou qualquer alteração nas características da córnea. Desta maneira, a possível ação isolada deste grupo quanto aos efeitos fotoquímicos não pode ser demonstrada. Em contrapartida, os dois grupos onde
Tabela 1

\section{Classificação das amostras estudadas após exposição das córneas porcinas após experimentação}

\begin{tabular}{lccccc}
\hline & $\boldsymbol{R B} 05+\boldsymbol{L}$ & $\boldsymbol{L}$ & $\boldsymbol{R B} 01+\boldsymbol{L}$ & $\boldsymbol{R B} \mathbf{0 1}$ & $\boldsymbol{R B} 05$ \\
\hline Amostra 1 & D & A & C & B & B \\
Amostra 2 & D & A & C & B & B \\
Amostra 3 & C & A & C & A & B \\
Amostra 4 & D & A & C & A & A \\
Amostra 5 & D & A & C & B & B \\
\hline
\end{tabular}

RB0,5 = Riboflavina 0,5\%; RB0,1 = Riboflavina $0,1 \% ; \quad$ L = LUZ.

foi empregado o uso de riboflavina irradiada, teve resultado positivo em nosso experimento, indo de encontro aos resultados já obtidos com os experimentos com luz ultravioleta.

Os grupos onde somente a riboflavina foi empregada demonstraram leve atividade do composto. Uma vez que a riboflavina pode sofrer decomposição, mesmo com luz branca, é esperado que uma reação fotoquímica frustrada possa ocorrer mesmo nessas condições. Outro fator que pode estar relacionado às alterações encontradas neste grupo,é o uso de dextran como veículo da solução de riboflavina. Sendo o dextran um agente hiperosmótico, uma pequena desidratação do estroma corneano poderia alterar as mudanças encontradas. Devemos ressaltar que essas diferenças não foram estatisticamente significantes.

Segundo os gráficos relativos à absorção da energia luminosa que foram anteriormente citados (Figura 2), existe uma relação da concentração de riboflavina e a absorção da energia luminosa. Este aumento na absorção da energia luminosa aumenta a degradação de riboflavina originando mais subprodutos oxidativos ${ }^{(12)}$.

Tabela 2

Resultados estatísticos das amostras utilizando teste de Kruskal-Wallis(comparação da diferença entre grupos utilizando teste de Bonferroni)

\begin{tabular}{lccccc}
\hline & $\begin{array}{c}\text { Riboflavina } \\
\mathbf{0 , 5} \%+\mathbf{L U Z}\end{array}$ & $\begin{array}{c}\text { Luz } \\
\text { somente }\end{array}$ & $\begin{array}{c}\text { Riboflavina } \\
\mathbf{0 , 1} \%+\mathbf{L U Z}\end{array}$ & $\begin{array}{c}\text { Riboflavina } \\
\mathbf{0 , 1} \%\end{array}$ & $\begin{array}{c}\text { Riboflavina } \\
\mathbf{0 , 5} \%\end{array}$ \\
\hline RB05 +L & - & $4,01^{*}$ & 0,89 & $3,01^{*}$ & 2,67 \\
L & $4,01^{*}$ & - & $3,12^{*}$ & 1 & 1,33 \\
RB01 +L & 0,89 & $3,12^{*}$ & - & 2,12 & 1,78 \\
RB01 & $3,01^{*}$ & 1 & 2,12 & - & 0,33 \\
RB05 & 2,67 & 1,33 & 1,78 & 0,33 & - \\
\hline
\end{tabular}

RB05 = Riboflavina 0,5\%; RB01 = Riboflavina $0,1 \% ; \quad L=$ LUZ

*Resultados baseados em valores de $\mathrm{Z}$ maiores que $2,81 \mathrm{com}$ significância estatística $(\mathrm{p}<0,05)$ 
O teste biomecânico aplicado em nosso trabalho, apesar de simples, corrobora os dados obtidos em outros trabalhos utilizando material não humano ${ }^{4}$. Por se tratar de um teste qualitativo, é recomendado que novos testes com utilização de dinanômetros seja aplicado a fim de comparar nossos resultados com trabalhos já publicados que utilizaram luz ultravioleta no processo fotoquímico ${ }^{(4)}$.

Possivelmente a presença de oxigênio atmosférico na experimentação diminuiu o efeito das reações covalentes que poderia ser potencializado com a experimentação em ambiente pobre em oxigênio ${ }^{(13)}$.

\section{Conclusão}

A utilização de riboflavina para efetuar o processo de aumento de ligações covalentes entre as fibrilas colágenas pode ser uma das chaves para o controle de doenças da córnea como o ceratocone. Nas concentrações estudadas e doses de irradiância concebidas, o processo de aumento das características biomecânicas da córnea foram obtidas com sucesso. Como era esperado, a concentração de riboflavina parece ter efeito sobre o resultado final nas ligações covalentes.

Novas pesquisas devem ser desenhadas para demonstrar a segurança, eficácia e doses máximas da medicação e irradiação. Mecanismos para minimizar a entrada de luz azul que atinge a retina, devem ser desenvolvidos para o sucesso desta opção terapêutica.

\section{Abstract}

Purpose: Demonstrate the possibility of changing the biomechanical behaviour of cornea stroma by usage of riboflavin associated with non-ultraviolet light. Methods: Double blind prospective study. Twenty five porcine eyes enucleated 24 hours before the experiment, have been divided on the following groups : Riboflavin 0,1\% with irradiation of blue light, Riboflavin 0,1\% without irradiation of light, Riboflavin 0,5\% with irradiation of blue light, Riboflavin 0,5\% without irradiation of light and BSS with irradiation of light. Results: Differences where noticed in groups 1 and $3(p<0,05)$. The groups without irradiation and the group with only irradiation, had no significant difference on their results. Conclusion: The usage of riboflavin appears to increase the crosslink connections among collagen fibrills. On the studied concentrations studied and radiant parameters the increase of biomechanical characteristics of cornea have been obtained successfully.
Keywords: Riboflavin/pharmacologia ; Photosensitizing agents/pharmacology; Corneal stroma/drug effects; Photochemistry; Biomechanics; Photobleaching; Ultraviolet rays; Swine

\section{RefERÊNCIAS}

1. Smelser GK, Polack FM, Ozanics V. Persistence of donor collagen in corneal transplants. Exp Eye Res. 1965; 4(4):349-54.

2. Spörl E, Schreiber J, Hellmund K, Seiler T, Knuschke P. [Untersuchungen zur Verfestigung der Hornhaut am Kaninchen]. Ophthalmologe. 2000; 97(3):203-6. German.

3. Wollensak G, Spoerl E, Seiler T. Stress-strain measurements of human and porcine corneas after riboflavin-ultraviolet-Ainduced cross-linking. J Cataract Refract Surg. 2003; 29(9):1780-5

4. Kohlhaas M, Spoerl E, Schilde T, Unger G, Wittig C, Pillunat LE. Biomechanical evidence of the distribution of cross-links in corneas treated with riboflavin and ultraviolet A light. J Cataract Refract Surg. 2006; 32(2):279-83.

5. Caporossi A, Baiocchi S, Mazzotta C, Traversi C, Caporossi T. Parasurgical therapy for keratoconus by riboflavin-ultraviolet type A rays induced cross-linking of corneal collagen: preliminary refractive results in an Italian study. J Cataract Refract Surg. 2006; 32(5):837-45. Comment in: J Cataract Refract Surg. 2007; 33(7):1143-4; author reply 1144.

6. Wollensak G, Wilsch M, Spoerl E, Seiler T. Collagen fiber diameter in the rabbit cornea after collagen crosslinking by riboflavin/UVA. Cornea. 2004; 23(5):503-7. Comment in: Cornea. 2004; 23(5):432.

7. Spoerl E, Wollensak G, Seiler T. Increased resistance of crosslinked cornea against enzymatic digestion. Curr Eye Res. 2004; 29(1):35-40.

8. Choe E, Min DB. Chemistry and reactions of reactive oxygen species in foods. Crit Rev Food Sci Nutr. 2006; 46(1):1-22.

9. Wollensak G, Spoerl E, Wilsch M, Seiler T. Keratocyte apoptosis after corneal collagen cross-linking using riboflavin/ UVA treatment. Cornea. 2004; 23(1):43-9.

10. Ahmad I, Fasihullah Q, Vaid FH. Effect of light intensity and wavelengths on photodegradation reactions of riboflavin in aqueous solution. J Photochem Photobiol B. 2006; 82(1): 21-7.

11. Paulino LV, Paulino E, Barros RA, Salles AG, Rehder JRCL. Alteração topográfica da curvatura corneana após aplicação de radiofreqüência em modelo animal. Arq Bras Oftalmol. 2005; 68(4):451-6.

12. Ahmad I, Fasihullah Q, Vaid FH. A study of simultaneous photolysis and photoaddition reactions of riboflavin in aqueous solution. J Photochem Photobiol B. 2004; 75(1-2):13-20.

13. Edwards AM, Bueno C, Saldaño A, Silva E, Kassab K, Polo L, Jori G. Photochemical and pharmacokinetic properties of selected flavins. J Photochem Photobiol B. 1999; 48(1):36-41.

\section{ENDEREÇO PARA CORRESPONDÊNCIA: \\ Rua Dom Gaspar Afonseca, 11 - apto 91 \\ CEP11045-360 - Santos - SP}

E-mail: gtg2001@terra.com.br 